\title{
CR Research Square \\ Primary Evaluation of Appling HMGA2 in the Diagnosis of Thyroid Carcinoma
}

\section{Jian Shen}

Shanghai Jiao Tong University Affiliated Sixth People's Hospital

Yi Li

Shanghai Jiao Tong University Affiliated Sixth People's Hospital

\section{Qiong Wu}

Shanghai Jiao Tong University Affiliated Sixth People's Hospital

\section{Yilun Liu}

Shanghai Jiao Tong University Affiliated Sixth People's Hospital

\section{Anqi Jin}

Shanghai Jiao Tong University Affiliated Sixth People's Hospital

\section{Bing $\mathrm{Hu}$}

Shanghai Jiao Tong University Affiliated Sixth People's Hospital

\section{Yan Wang ( $\nabla$ yannanfly@126.com )}

Shanghai Jiaotong University Affiliated Sixth People's Hospital https://orcid.org/0000-0003-1675-9065

\section{Research article}

Keywords: High mobility group protein A2 (HMGA2), Thyroid carcinoma, Cervical lymph node metastasis, Immunohistochemistry

Posted Date: May 18th, 2021

DOI: https://doi.org/10.21203/rs.3.rs-531082/v1

License: (c) (1) This work is licensed under a Creative Commons Attribution 4.0 International License. Read Full License 


\section{Abstract \\ Background}

Molecular marker is on the hot spot for thyroid tumor research in the recent years. The purpose of this study is try to clarify the value of HMGA2 for differential diagnosis between benign and malignant thyroid nodules in order to further assessing the risk of simultaneous cervical lymph nodes metastasis.

\section{Methods}

With a total of 125 thyroid samples, including 85 papillary thyroid carcinomas, 20 follicular thyroid adenomas and 20 normal thyroid tissue were retrospectively analyzed, and we compare expression level of HMGA2 protein among these three groups in this study. PTC group was then subdivided according to the number of metastasis lymph nodes in order to explore the possibility of using HMGA2 protein to predict invasion of lymph nodes in PTC. We also compare the efficiency of BRAF ${ }_{\mathrm{V} 600 \mathrm{E}}$ mutation and HMGA2 expression level in the prediagnosis of cervical lymph node metastasis during the experiment.

\section{Results}

Expression rate of HMGA2 protein in PTC was measured at 100\%, which has significant statistical differences with HMGA2 in FTA and NT $(P<0.05)$. Moreover, there was no significant correlation between expression level of HMGA2 and the size of PTC; whether it accompanied by Hashimoto's thyroiditis; high expression level of HMGA2 could be more sensitive in the prediagnosis of cervical lymph node metastasis than BRAF ${ }_{\mathrm{V} 600 \mathrm{E}}$ mutation.

\section{Conclusion}

\HMGA2 protein can be used as a molecular marker for differential diagnosis of benign and malignant thyroid nodules. $\triangle$ The expression level of HMGA2 protein is correlated with cervical lymph node metastasis in papillary thyroid carcinoma, performing an auxiliary value for clinical decision.

\section{Background}

Thyroid carcinoma, most common endocrine malignancies, is also the fastest growing malignant tumor in the world[1, 2]. High-resolution ultrasound which considered as the most effective method for detecting diagnosing thyroid carcinoma[3]. Studies have shown about 19 to 67 percent of people were diagnosed with thyroid nodules by high-frequency ultrasound among 7 to 15 percent was malignant[4,5]. Currently, percutaneous fine needle aspiration (FNA) cytology, considered as the best practice for the management 
of thyroid nodules. However combining both two approaches together, they still fail to provide definitive malignancy confirmation of a thyroid nodule in many cases [6].

Operation is the first choice for thyroid carcinoma; however, due to limited preoperative diagnosis, some patients with cervical lymph nodes metastasis are being miss diagnosed. This is also the reason that the treatment of papillary thyroid micro-carcinoma(PTMC) has always been controversial. Some doctors believe patients with PTMC can be monitored on a regular basis; others point out that although some of the PTMC can reach as small as $5 \mathrm{~mm}$, it could still occur with imperceptible lymph nodes metastasis, requiring early clinical intervention [7]. Therefore, preoperative evaluation posts great importance to both patients and surgeons.

Accurate molecular markers for preoperative diagnosis of thyroid carcinomas could provide patients with reasonable treatment options; high mobility group protein AT-hook 2 (HMGA2) gene plays an important role of fetal development and tumorigenesis. And overexpression of HMGA2 has been reported as a feature of many malignant tumors of epithelial origin, it also associated with poor prognosis, such as chemoresistance, metastases and low long-survival rate[8-10].

Herein, we choose HMGA2 to be the marker to evaluate potential of its expression level in the diagnosis and prognosis of thyroid nodules.

\section{Methods}

\section{Subjects}

Patients enrolled with thyroid nodules admitted to our center from August 2016 to August 2017. Total of 125 samples were randomly selected, 85 cases of papillary thyroid carcinoma(PTC), 20 cases of follicular thyroid adenoma(FTA) and 20 cases of normal thyroid tissue(NT). Enrollment criteria: (1) confirmed pathological diagnosis; (2) thyroid ultrasound examination and surgery were performed in our hospital and all the information were complete; (3) One patient corresponds to one pathological type. Exclusion criteria: (1) uncertain pathological diagnosis; (2) no obvious thyroid nodules; (3) Cervical lymph nodes removal not performed. (4) History of thyroid carcinoma of other pathological type except PTC; (5) History of radiotherapy, chemotherapy or other thyroid-related interventions.

This study receive approval from the Ethical Committee of Shanghai Jiao Tong University Affiliated Sixth People's Hospital. All procedures performed in the study involving human participants were in accordance with the ethical standards of the institutional research committee and the declaration of Helsinki [11].

\section{Intraoperative tissue acquisition}

All samples for this study, including PTC, FTA and NT, were obtained intraoperatively, tissues of PTC/FTA were taken from the center part of the thyroid nodules. NT was taken from the part with a minimum of $1 \mathrm{~cm}$ away from the margin of tumor. Extreme cases such as tumor can occupy most of gland, forcing 
NT taken from the opposite side. After all tissues were put in the sterile tubes, then moved to $-80^{\circ} \mathrm{C}$ immediately for future use; sample tissue size taken from the experiment measured across 4 to $5 \mathrm{~mm}$.

\section{Immunohistochemical analysis of clinical samples}

Process start with defrosting the tissue samples under room temperature, then adding formalin and setting for 24 hours for preparing the paraffin-embedded tissue. Six- $\mu$ m-thick paraffin sections were serially cut from paraffin-embedded tissues; sections obtained mounted onto slides; and then deparaffinized. All slides were rinsed in PBS for three 5-minute cycles, and then methanol containing $0.3 \%$ hydrogen peroxide was added to the slides. After incubating for $20 \mathrm{~min}$ at $37^{\circ} \mathrm{C}$, slides were rinsed in distilled water for another three 5-minute cycles.

For immunohistochemical detection of HMGA2, slides were incubated in a humid chamber at $37^{\circ} \mathrm{C}$ with 1:100 dilution of rabbit anti-human HMGA2 polyclonal (Protein Group, Inc., Wuhan, China) antibody. Bound antibodies (Protein Group, Inc., Wuhan, China) were added subsequently follow by incubation in a humid chamber at $37^{\circ} \mathrm{C}$ for $30 \mathrm{~min}$.

We used diaminobenzidine as the chromogen in this experiment. After the expected stain intensity developed, we rinsed slides thoroughly by running water to stop color rendering; sections were lightly counterstained with hematoxylin in the experiment. Given negative controls, immunostaining was performed by incubating samples with PBS instead of the primary antibody; remaining steps can be duplicated from previous procedures.

\section{Immunohistochemical evaluation}

Cells marked positive for HMGA2 when immunoreactivity was observed in either cytoplasm or nuclei. The immunohistochemical results of tissue samples with no positive cells considered negative, those with positive cells were positive; especially for those with cytoplasm and nuclei simultaneously positive as strongly positive.

\section{Statistical analysis}

Imaging acquisition was taken by Olympus BX53 Research Biomicroscope (Olympus Co., Japan). Analyzing the captured images (SP $\times 400)$ by Motic Med 6.0 Digital Medical Imaging System (Motic china group Co., Ltd, Xiamen, China). The average optical density value (AOD) of 10 image-positive cell staining was chosen as a reference indicator for the intensity of the immunohistochemistry response, indicating the amount of antigen expression.

Data are presented with mean \pm SD and analyzed with SPSS 24.0, T-test and ANOVA was used as appropriate. If there is no special explanation, $p<0.05$ was considered statistically significant.

\section{Results}

\section{Population characteristics}


Total of 125 thyroid tissues, including 85 PTC, 20 FTA and 20 NT, were analyzed in this study, among 125 patients, $58.4 \%(73 / 125)$ were females and $41.5 \%(52 / 125)$ were males; the average age of the patients was 44 years old (range from 15 to 80 yrs.). 40 patients, including 5 NT, 13 FTA and 22 PTC, were simultaneously diagnosed as Hashimoto's thyroiditis(HT). 85 cases of PTC consisted 36 PTMC and 49 non-PTMC. The number of 85 PTC patients with or without BRAF ${ }_{\mathrm{V} 600 \mathrm{E}}$ mutation was 48 and 37 , respectively (Table 1$)$.

Table 1

Patient Characteristics

\begin{tabular}{|c|c|c|c|c|}
\hline \multicolumn{2}{|l|}{ Group } & \multirow{2}{*}{$\begin{array}{l}\text { Normal } \\
(n=20) \\
8\end{array}$} & \multirow{2}{*}{$\begin{array}{l}\text { FTA } \\
(n=20) \\
12\end{array}$} & \multirow{2}{*}{$\begin{array}{l}\text { PTC } \\
(n=85) \\
53\end{array}$} \\
\hline Sex & Female & & & \\
\hline & Male & 12 & 8 & 32 \\
\hline \multirow[t]{2}{*}{ Age } & & $42 \pm 12$ & $52 \pm 11$ & $40 \pm 14$ \\
\hline & & $(22 \sim 66)$ & $(33 \sim 70)$ & $(15 \sim 80)$ \\
\hline \multirow[t]{2}{*}{ Size of nodules } & $\leq 1 \mathrm{~cm}$ & - & - & 36 \\
\hline & $\nabla 1 \mathrm{~cm}$ & - & - & 49 \\
\hline \multirow[t]{2}{*}{ HT } & $(+)$ & 5 & 13 & 22 \\
\hline & $(-)$ & 15 & 7 & 63 \\
\hline \multirow{2}{*}{$\begin{array}{l}\text { BRAF }_{\mathrm{V} 600 \mathrm{E}} \\
\text { mutation }\end{array}$} & $(+)$ & - & - & 48 \\
\hline & $(-)$ & - & - & 37 \\
\hline
\end{tabular}

\section{Expression of HMGA2 protein in different thyroid tissue}

All tissues were confirmed by two pathologists respectively after H\&E stained. 15(17.6\%) PTC from 85 cases was selected randomly to compare the differential expression of HMGA2 protein with 20 FTA and 20 NT by IHC. Through the preliminary observation, result showed HMGA2 was negative in NT; optical microscope showed the nucleus and cytoplasm were not significantly stained (Fig. 1A). HMGA2 in FTA was weak positive; optical microscope showed the nucleus was light brown stained and the cytoplasm was not stained (Fig. 1B). HMGA2 in PTC was strongly positive; optical microscope showed the nucleus was deep brown stained, the cytoplasm was brownish yellow stained (Fig. 1C).

The expression of HMGA2 among three groups is significantly different by semi-quantitative method, $p<$ 0.01 (NT vs FTA $p<0.01$; NT vs PTC $p<0.01$; FTA vs PTC $p<0.01$ ). AOD of PTC was $0.53329 \pm$ 0.01131 (95\%Cl: $0.50903,0.55755)$, FTA was $0.21584 \pm 0.00720(95 \% \mathrm{Cl}: 0.20076,0.23091)$ and NT was 
$0.08025 \pm 0.00105$ (95\% Cl: 0.07805.0.08245) (Fig. 2A); further analysis suggested that expression of HMGA2 protein was independent from tumor size $(p=0.013,|r|=0.268)$. After dividing the PTC group into PTMC and non-PTMC subgroups, the expression level of HMGA2 protein was compared between two groups; AOD of PTMC was $0.50987 \pm 0.04260$ (95\% Cl: $0.49546,0.52429)$, and non-PTMC was $0.51294 \pm$ 0.04510 (95\% Cl: $0.49999,0.52590)$. Results showed there was no statistical difference between the two groups $(p=0.750)($ Fig. 2B).

Then we compared the expression level of HMGA2 protein in PTC with or without HT, result showed there was no significant difference between the two groups $(p=0.564)$. Statistical results also showed that the expression of HMGA2 was independent of patients' gender(pख0.05).

Furthermore, we analyze the correlation between expression of HMGA2 and cervical lymph nodes metastasis in PTC patients and found out AOD of HMGA2 in patients with or without cervical lymph nodes metastasis was $0.52240 \pm 0.00563(95 \% \mathrm{Cl}: 0.51112,0.53368), 0.48582 \pm 0.00649,(95 \% \mathrm{Cl}$ : $0.47242,0.49923)$, respectively. Result showed there was statistical difference between above two groups $(p=0.000)$ (Fig. 2C); the area under the ROC curve (AUC) measured $0.737(p<0.05)$, indicated the expression level of HMGA2 was statistically significant in determining whether PTC has cervical lymph node metastasis. Cutoff value of AOD was 0.51 (Fig. 3).

Comparison of HMGA2 expression with BRAF ${ }_{\text {V600E }}$ mutation to judge the diagnostic efficacy of cervical lymph node metastasis in PTC

Our study concluded $56.57 \%$ (48/85) PTC accompanied by BRAF $\mathrm{V}_{600 \mathrm{E}}$ mutation. 38 out of 60 patients who suffered cervical lymph node metastasis had BRAF ${ }_{\mathrm{V} 600 \mathrm{E}}$ mutation; 10 out of 25 patients without cervical lymph node metastasis had BRAF ${ }_{\mathrm{V} 600 \mathrm{E}}$ mutation. Sensitivity of BRAF $\mathrm{V}_{\mathrm{V} 600 \mathrm{E}}$ mutation to indicate cervical lymph nodes was $79.17 \%$, specificity was $40.54 \%$, positive prediction was $63.33 \%$, negative prediction was $60 \%$, false positive rate was $59.46 \%$, and false negative rate was $20.83 \%$. We obtained Cutoff $_{A O D}=0.51$ in our earlier study, so we hypothesized that $A O D>0.51$ indicated cervical lymph node metastasis, while AOD $<0.51$ indicated no lymph node metastasis. The sensitivity result came $89.47 \%$, specificity was $44.68 \%$, positive prediction was $56.67 \%$, negative prediction was $84 \%$, false positive rate was $55.32 \%$, false negative rate was $10.53 \%$ (Table 2 and Table 3 ). 
Table 2

Comparison of HMGA2 and BRAF $\mathrm{V}_{\mathrm{V} 600 \mathrm{E}}$ in predicting the metastasis of cervical lymph nodes in PTC

\begin{tabular}{|c|c|c|c|c|c|c|}
\hline \multirow[t]{2}{*}{ Lymph node metastasis } & \multicolumn{2}{|c|}{ BRAF $_{\mathrm{V} 600 \mathrm{E}}$ mutation } & \multirow[t]{2}{*}{ Sum } & \multicolumn{2}{|c|}{ HMGA2 expression level* } & \multirow[t]{2}{*}{ Sum } \\
\hline & $(+)$ & $(-)$ & & $(+)$ & $(-)$ & \\
\hline$(+)$ & 38 & 22 & 60 & 34 & 26 & 60 \\
\hline$(-)$ & 10 & 15 & 25 & 4 & 21 & 25 \\
\hline Sum & 48 & 37 & 85 & 38 & 47 & 85 \\
\hline
\end{tabular}

Table 3

Comparison of HMGA2 and BRAF $\mathrm{V}_{600 \mathrm{E}}$ in predicting the metastasis of cervical lymph nodes in PTC

\begin{tabular}{|c|c|c|c|c|c|c|}
\hline & SEN & SPE & PPV & NPV & FPR & FNR \\
\hline BRAF $_{\mathrm{V} 600 \mathrm{E}}$ mutation* & $79.17 \%$ & $40.54 \%$ & $63.33 \%$ & $60.00 \%$ & $59.46 \%$ & $20.83 \%$ \\
\hline HMGA2 expression level ${ }^{\star \star}$ & $89.47 \%$ & $44.68 \%$ & $56.67 \%$ & $84.00 \%$ & $55.32 \%$ & $10.53 \%$ \\
\hline \multicolumn{7}{|c|}{$\rrbracket:{ }^{*} \mathrm{BRAF}_{\mathrm{V} 600 \mathrm{E}}$ mutation,positive or negative. } \\
\hline \multicolumn{7}{|c|}{ 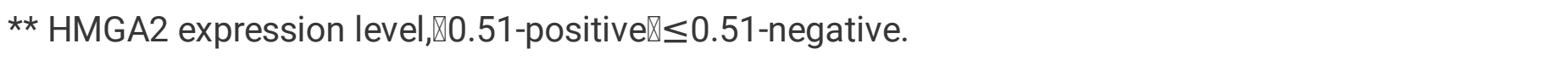 } \\
\hline \multicolumn{7}{|c|}{$\begin{array}{l}\text { SEN-sensitivity, SPE-specificity } \square P P V \text {-positive prediction } \square N P V \text {-negative prediction } \square F P R \text {-false positive } \\
\text { rate[FNR-false negative rate. }\end{array}$} \\
\hline
\end{tabular}

\section{Discussion}

Most PTC are inert growth with good prognosis afterwards, but the probability of cervical lymph node metastasis, which was not detected by ultrasound before surgery, is still existed with the rate of $20 \%-30 \%$ $[12,13]$. Wang et al. confirmed that age $(<55 \mathrm{yrs}$.), male, tumor size $(0.5-1.0 \mathrm{~cm})$, multifocal are risk factors for predicting cervical lymph node metastasis in the central region of neck in PTMC patients [14]. However, lymph nodes metastasis in central region still can be easily missed during ultrasound scanning. Previous studies have found the specificity of preoperative ultrasound detection of cervical lymph node metastasis in PTC patients was $87 \%$ 97\%, but the sensitivity measure was only $23 \% \sim 38 \%$ [15]. Since the involvement of lymph nodes greatly affects the clinical treatment strategy, it is helpful to assess the risk of lymph node metastasis in patients by combining preoperative ultrasound and molecular biological indicators.

High mobility family protein A2 (HMGA2), previously known as HMGI-C, was firstly discovered by Giancotti et al as a nuclear protein associated with malignant phenotype of rat thyroid cells transformed with murine retroviruses in 1985[16]. While the expression of HMGA2 could be tested in several benign 
mesenchymal tumors, most predominantly lipomas, was mainly found in malignant tumors; HMGA2 would be present at a much higher level in malignancy, such as prostate cancer, ovarian cancer, gastric cancer, gallbladder cancer, breast cancer, and etc. [8, 9, 17-19]. Previous studies have demonstrated that malignant tumors expressing HMGA2 usually come up with a poor prognosis [10, 20-24], thus, HMGA2 possess the potential for diagnosis of thyroid malignancies.

After experiment, we confirmed that the expression level of HMGA2 in PTC is significantly higher than FTA and NT cases $(85 / 85,100 \%)$. In addition, we found that a small amount of HMGA2 protein expression in NT(AOD $=0.08084 \pm 0.00472)$, of which the expression level could be negligible. We speculate that the reason may be associated with microcirculation, since the normal thyroid tissue we obtained essentially from the area $1 \mathrm{~cm}$ away from the nodules in the ipsilateral thyroid. We analyzed the correlation between PTC and expression of HMGA2 protein and found that HMGA2 expression did not show significantly correlation with neither the size of PTC nor the gender of patients, and there was no statistical difference for the expression of HMGA2 between the PTMC and non-PTMC. We also proved that Hashimoto's thyroiditis had no impact on the expression level of HMGA2 protein in PTC, which has not been reported in previous studies.

We found there is statistical difference between the two groups by comparing the expression of HMGA2 of patients with or without cervical lymph nodes metastasis. According to the ROC curve, we calculated the cutoff value at 0.51 , which means if AOD of HMGA2 went over 0.51 , there was a high probability that the patient suffered lymph nodes metastasis with a sensitivity of $89.47 \%$ and specificity of $44.68 \%$.

Results from this research show a significant increase of the possibility to predict the prognosis of PTC and a supplementary to the choice of clinical treatment.

Previous studies showed BRAF ${ }_{\mathrm{V} 600 \mathrm{E}}$ is the most common genetic mutation in PTC, associated with an increased ability of lymph nodes metastasis[6]. According to previous studies, the rate of $\mathrm{BRAF}_{\mathrm{V} 600 \mathrm{E}}$ mutation in classical PTC was $28.4 \% \sim 75.3 \%$; however, there are some limitations in clinical application because of its low sensitivity $[25,26]$. We compared the diagnostic efficiency in the judgment of the cervical lymph nodes aggression between $\mathrm{HMGA} 2$ expression level and BRAF ${ }_{\mathrm{V} 600 \mathrm{E}}$ mutation during our study. Result suggested that HMGA2 was more sensitive than $\mathrm{BRAF}_{\mathrm{V} 600 \mathrm{E}}\left(\chi^{2}=5.831, p=0.016 \square \mathrm{SEN}\right.$ $89.47 \%$ vs $79.17 \%$ ), and false positive rate and false negative rate of HMGA2 were slightly lower than BRAF $_{\text {V600E }}$ (FPR 55.32\% vs 59.46\%, FNR 10.53\% vs $20.83 \%$ ). We conclude all results with the expression level of HMGA2 protein has a relatively better diagnostic effect than the BRAF ${ }_{\mathrm{V} 600 \mathrm{E}}$ mutation for the judgment of cervical lymph nodes metastasis.

\section{Conclusion}

In summary, this study demonstrates the differential diagnostic value of HMGA2 protein expression for thyroid nodules, and we confirm that HMGA2 protein is associated with cervical lymph node metastasis in PTC. Therefore, we could assess the risk of cervical lymph node metastasis based on the expression level of HMGA2. Thus, HMGA2 could to be a new molecular marker for the diagnosis and prognosis of 
thyroid tumors. Furthermore, when combined with FNA, it may provide preoperative evaluation for patients better than BRAF. In order to explore the convincement of our results, we will further perform prospective clinical trials.

\section{Abbreviations}

HMGA2: High mobility group protein AT-hook 2; IHC: Immunohistochemistry; AOD: Average optical density; FNA: Fine needle aspiration; US: Ultrasound; FTA: Follicular thyroid adenoma. PTC: Papillary thyroid carcinoma. HT: Hashimoto's thyroiditis; AUC: Area under the curve; Cl: Confidence internal.

\section{Declarations}

\section{Ethics approval and consent to participate}

This study was approved by Ethics Committee of Shanghai Sixth People's Hospital. All procedures performed in the study involving human participants were in accordance with the ethical standards of the institutional research committee and the 1964 Helsinki declaration and its later amendments or comparable ethical standards. All patients provided informed consent to participate in the study.

\section{Consent for publication}

All participants agreed with using individual data under anonymity.

\section{Available of data and materials}

The dataset used and analyzed during the current study are included in this published article.

\section{Competing interest}

The authors declare that they have no competing interest.

\section{Funding}

This work was supported by National Natural Science Foundation of China (Grant No.81671700 and No.81701706), Shanghai key discipline of medical imaging (Grant No.2017 ZZ 02005), and Shanghai Key Clinical Disciplines Fund (Grant No. shslczdzk03203).

\section{Author's contribution}

JS, YL, YW contributed to the conception and design of the study. All the US images in this article were provided by prof. YW. JS, QW, YL and QJ performed for subsequent trials including sample collection, IHC and data analysis. JS wrote the manuscript. YW and $\mathrm{BH}$ revised the manuscript. All authors reviewed and approved the final version of manuscript. 
Acknowledgments

We acknowledgement Mr. Johnson Sheng for reviewing the manuscript for grammar consistency.

\section{References}

1. Disease GBD, Injury I, Prevalence C. Global, regional, and national incidence, prevalence, and years lived with disability for 328 diseases and injuries for 195 countries, 1990-2016: a systematic analysis for the Global Burden of Disease Study 2016. Lancet (London, England). 2017;390(10100):1211-59.

2. Carling T, Udelsman R. Thyroid cancer. Annu Rev Med. 2014;65:125-37.

3. Bojunga J. Ultrasound of Thyroid Nodules. Ultraschall Med (Stuttgart, Germany : 1980). 2018;39(5):488-511.

4. Haugen BR, Alexander EK, Bible KC, Doherty GM, Mandel SJ, Nikiforov YE, et al. 2015 American Thyroid Association Management Guidelines for Adult Patients with Thyroid Nodules and Differentiated Thyroid Cancer: The American Thyroid Association Guidelines Task Force on Thyroid Nodules and Differentiated Thyroid Cancer. Thyroid. 2016;26(1):1-133.

5. Tan GH, Gharib H. Thyroid incidentalomas: management approaches to nonpalpable nodules discovered incidentally on thyroid imaging. Ann Intern Med. 1997;126(3):226-31.

6. Albarel F, Conte-Devolx B, Oliver C. From nodule to differentiated thyroid carcinoma: contributions of molecular analysis in 2012. Ann Endocrinol. 2012;73(3):155-64.

7. Ito Y, Miyauchi A, Kihara M, Higashiyama T, Kobayashi K, Miya A. Patient age is significantly related to the progression of papillary microcarcinoma of the thyroid under observation. Thyroid. 2014;24(1):27-34.

8. Morishita A, Zaidi MR, Mitoro A, Sankarasharma D, Szabolcs M, Okada Y, et al. HMGA2 is a driver of tumor metastasis. Cancer Res. 2013;73(14):4289-99.

9. Wu J, Zhang S, Shan J, Hu Z, Liu X, Chen L, et al. Elevated HMGA2 expression is associated with cancer aggressiveness and predicts poor outcome in breast cancer. Cancer Lett. 2016;376(2):284-92.

10. Na N, Si T, Huang Z, Miao B, Hong L, Li H, et al. High expression of HMGA2 predicts poor survival in patients with clear cell renal cell carcinoma. OncoTargets Ther. 2016;9:7199-205.

11. World Medical Association World Medical Association Declaration of Helsinki-ethical principles for medical research involving human subjects. 2013. Available at: www.wma.net/policies-post/wmadeclaration-of-helsinki-ethical-principles-for-medical-research-involving-human-subjects/. Accessed July 31, 2017.

12. Wei T, Chen R, Zou X, Liu F, Li Z, Zhu J. Predictive factors of contralateral paratracheal lymph node metastasis in unilateral papillary thyroid carcinoma. Eur J Surg Onc. 2015;41(6):746-50.

13. Bhatia KS, Lee YY, Yuen EH, Ahuja AT. Ultrasound elastography in the head and neck. Part II. Accuracy for malignancy. Cancer Imaging. 2013;13(2):260-76. 
14. Wang Y, Guan Q, Xiang J. Nomogram for predicting central lymph node metastasis in papillary thyroid microcarcinoma: A retrospective cohort study of 8668 patients. Int J Surg. 2018;55:98-102.

15. T KM, M DK, B SR, N AN, R PL, D BM, et al. Preoperative High-Resolution Ultrasound for the Assessment of Malignant Central Compartment Lymph Nodes in Papillary Thyroid Cancer. Thyroid. 2015(12).

16. Giancotti V, Berlingieri MT, DiFiore PP, Fusco A, Vecchio G, Crane-Robinson C. Changes in nuclear proteins on transformation of rat epithelial thyroid cells by a murine sarcoma retrovirus. Cancer Res. 1985;45(12 Pt 1):6051-7.

17. Grünewald I, Trautmann M, Busch A, Bauer L, Huss S, Schweinshaupt P, et al. MDM2 and CDK4 amplifications are rare events in salivary duct carcinomas. Oncotarget. 2016;7(46):75261-72.

18. Mahajan A, Liu Z, Gellert L, Zou X, Yang G, Lee P, et al. HMGA2: a biomarker significantly overexpressed in high-grade ovarian serous carcinoma. Modern pathol. 2010;23(5):673-81.

19. Dong J, Wang R, Ren G, Li X, Wang J, Sun Y, et al. HMGA2-FOXL2 Axis Regulates Metastases and Epithelial-to-Mesenchymal Transition of Chemoresistant Gastric Cancer. Clin Cancer Res. 2017;23(13):3461-73.

20. Lee CT, Wu TT, Lohse CM, Zhang L. High-mobility group AT-hook 2: An independent marker of poor prognosis in intrahepatic cholangiocarcinoma. Hum Pathol. 2014;45(11):2334-40.

21. Wu J, Zhang S, Shan J, Hu Z, Liu X, Chen L, et al. Elevated HMGA2 expression is associated with cancer aggressiveness and predicts poor outcome in breast cancer. Cancer Lett. 2016;376(2):284-92.

22. Singh I, Mehta A, Contreras A, Boettger T, Carraro G, Wheeler M, et al. Hmga2 is required for canonical WNT signaling during lung development. BMC Biol. 2014;12:21.

23. Liu TP, Huang CC, Yeh KT, Ke TW, Wei PL, Yang JR, et al. Down-regulation of let-7a-5p predicts lymph node metastasis and prognosis in colorectal cancer: Implications for chemotherapy. Surg Oncol. 2016;25(4):429-34.

24. Chang KP, Lin SJ, Liu SC, Yi JS, Chien KY, Chi LM, et al. Low-molecular-mass secretome profiling identifies HMGA2 and MIF as prognostic biomarkers for oral cavity squamous cell carcinoma. Sci Rep. 2015;5:11689.

25. Liu L, Chang JW, Jung SN, Park HS, Oh T, Lim YC, et al. Clinical implications of the extent of BRAF(V600E) alleles in patients with papillary thyroid carcinoma. Oral Oncol. 2016;62:72-7.

26. Sugino K, Nagahama M, Kitagawa W, Shibuya H, Ohkuwa K, Uruno T, et al. Papillary Thyroid Carcinoma in Children and Adolescents: Long-Term Follow-Up and Clinical Characteristics. World J Surg. 2015;39(9):2259-65.

\section{Figures}




\section{A}
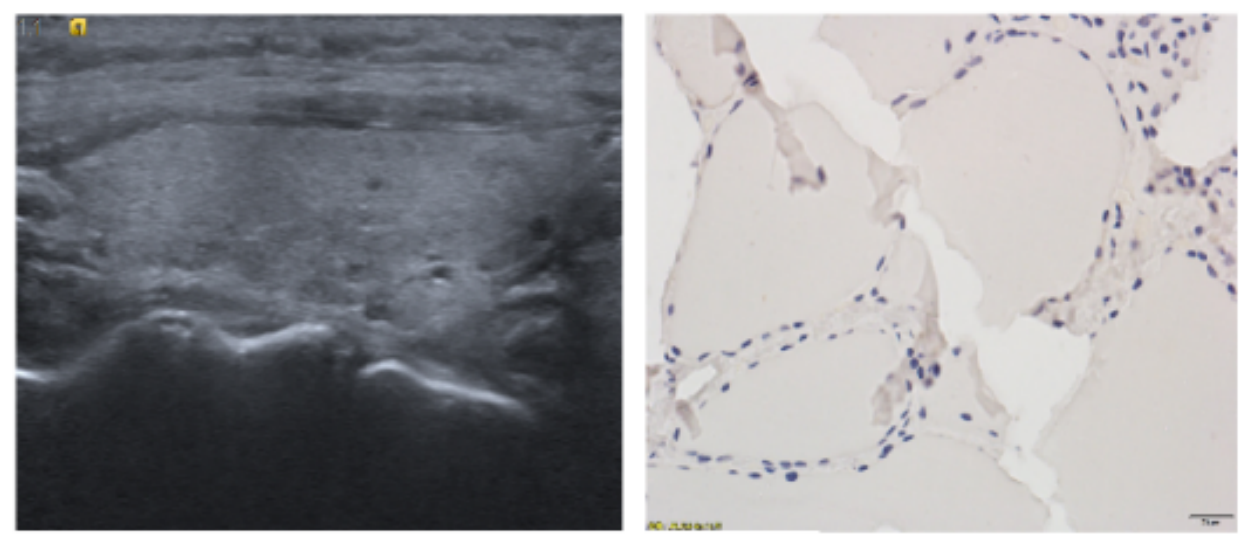

B
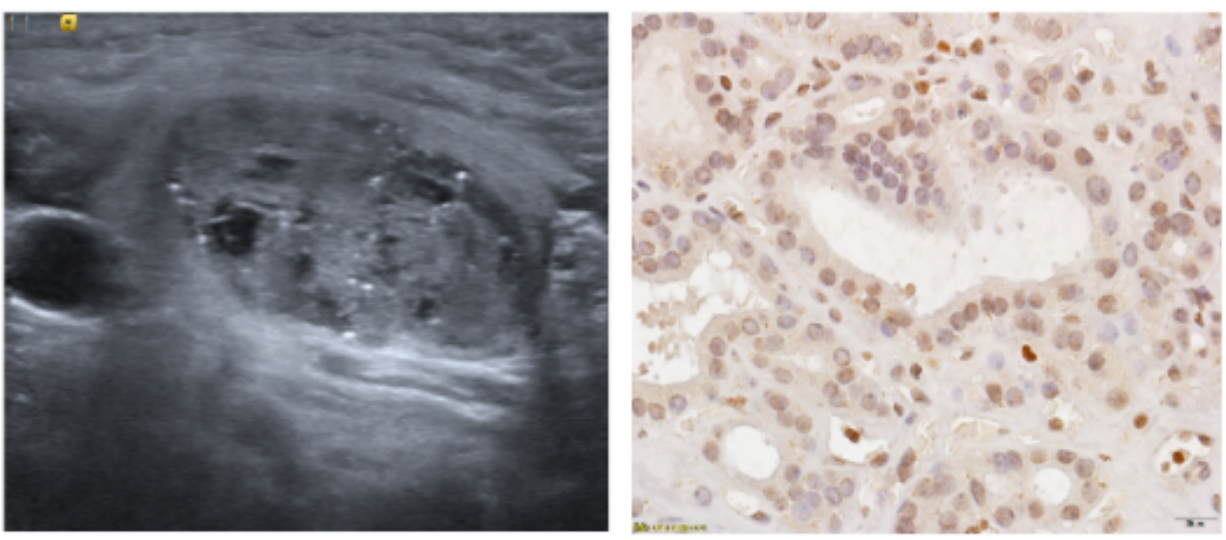

C
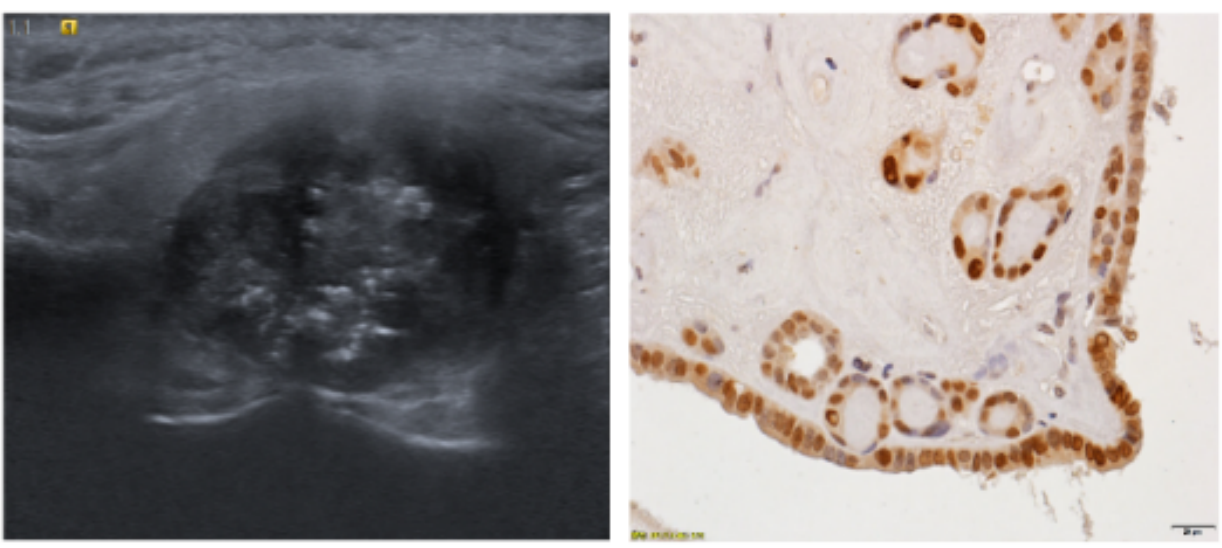

\section{Figure 1}

Ultrasound and immunohistochemistry results of HMGA2 in different thyroid tissue. A. Normal thyroid

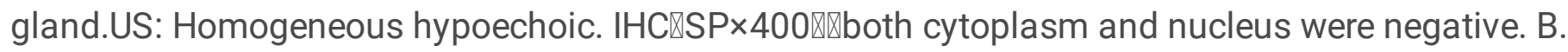

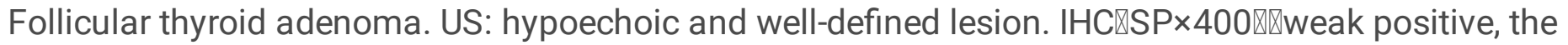
nucleus was light brown stained and the cytoplasm was not stained. C. Papillary thyroid carcinoma. US: 
hypoechoic lesion with obscure boundary and calcification. IHC $\triangle S P \times 400 \otimes \Downarrow$ strongly positive, the nucleus was deep brown stained, the cytoplasm was brownish yellow stained.
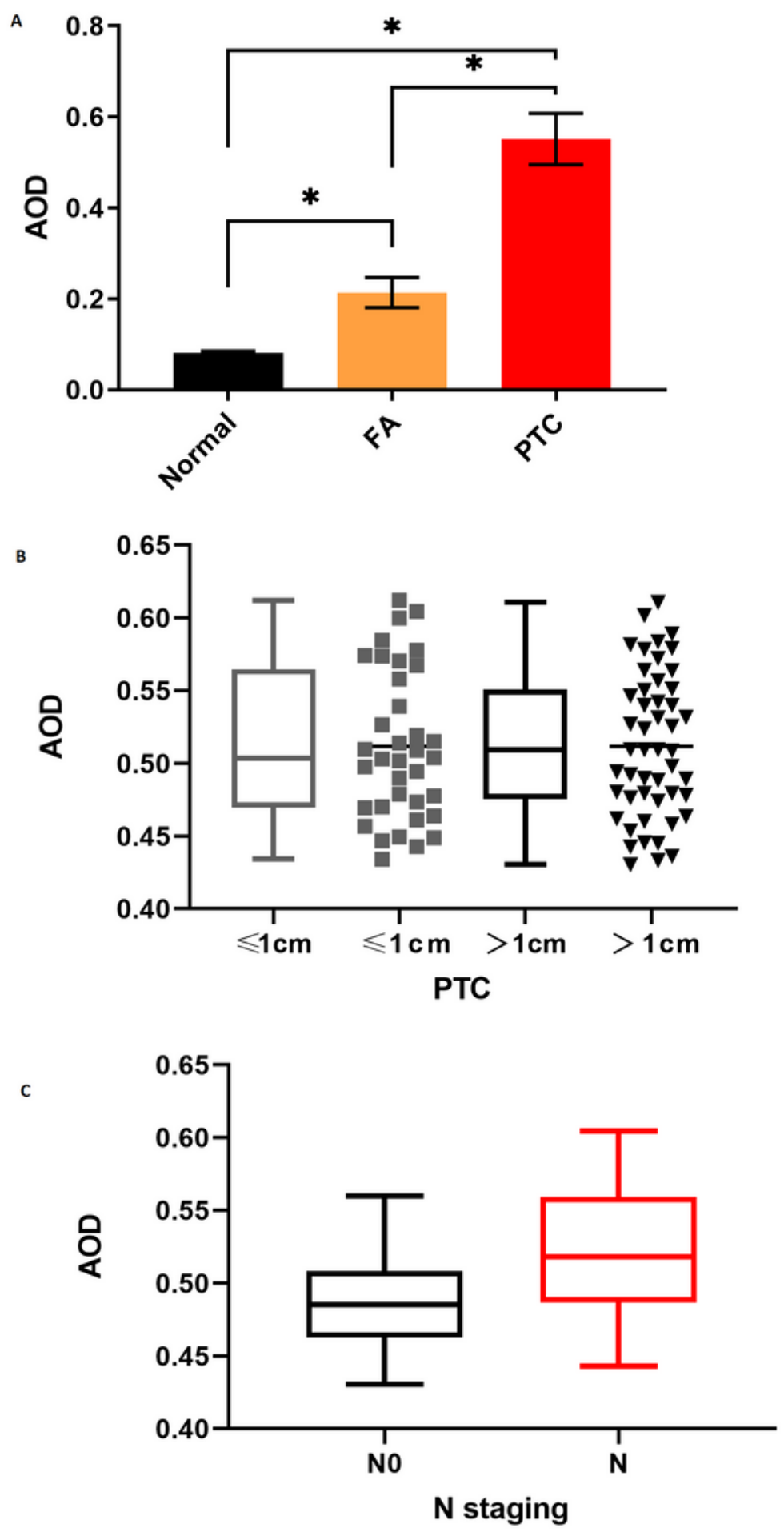

Figure 2

A. Comparison of AOD among Normal group, FTA group and PTC group. There were statistical differences among the three groups by pairwise comparison $(P<0.01)$. B. There was no statistical difference in the expression level of HMGA2 in PTMC and non-PTMC $(P>0.05)$. C. The expression of 
HMGA2 in PTC with or without cervical lymph nodes metastasis. There was statistical difference between two groups, $\mathrm{p}(\mathrm{N} 0$ vs $\mathrm{N}) \otimes 0.05$. N0-no evidence of regional lymph nodes metastasis; $\mathrm{N}$ - cervical lymph nodes metastasis.

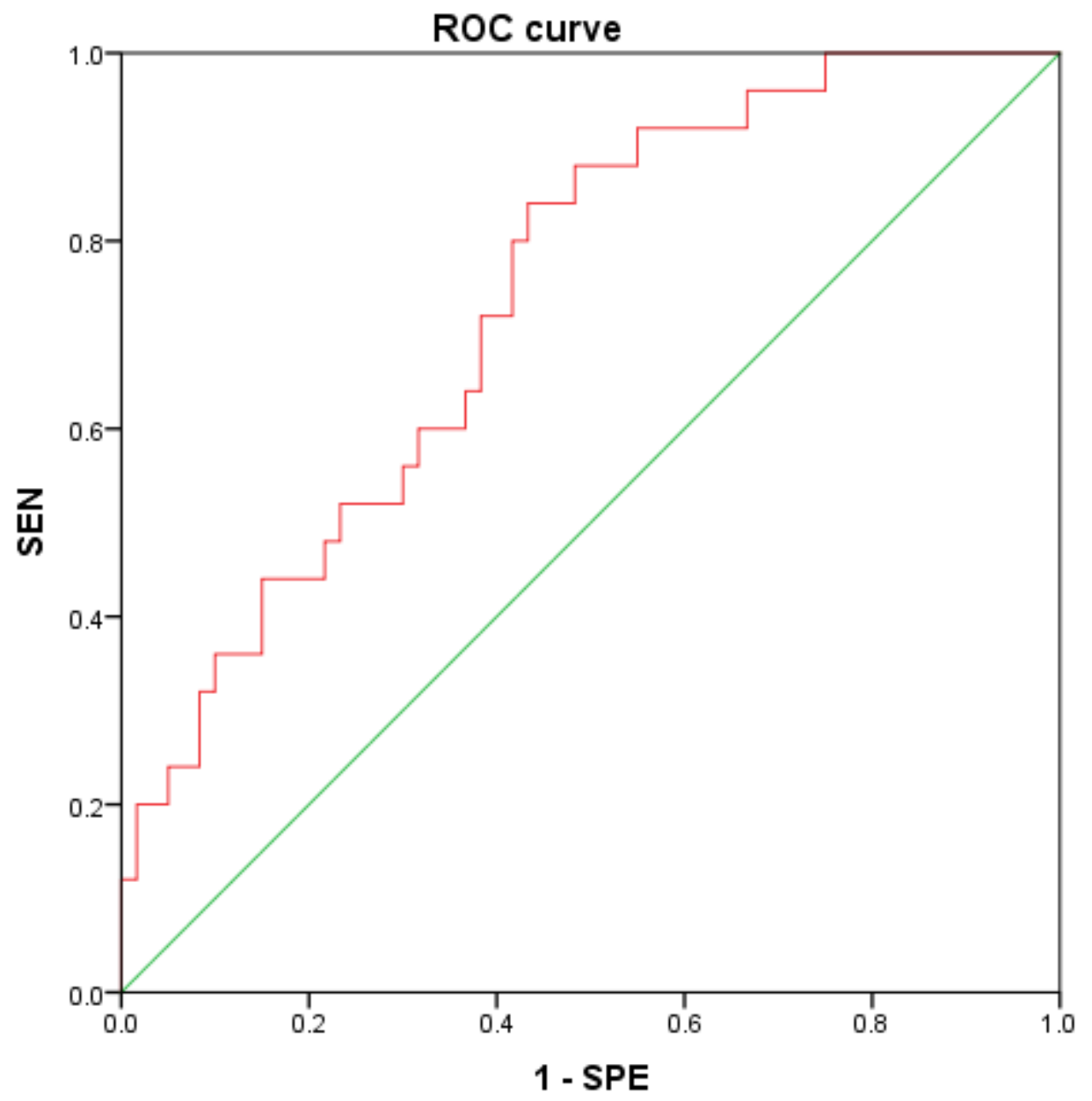

Figure 3

ROC curve. The ROC curve was plotted by the presence/absence of cervical lymph nodes as state variables. AUC (Area under the curve) $=0.737, p=0.001$. 